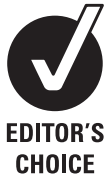

CHOICE
See Editorial, p 189

- An additional supplement is published online only. To view this file please visit the journal online (http://thorax.bmj.com/ content/67/3.toc)

${ }^{1}$ Centre for Asthma and Respiratory Disease, The University of Newcastle, School of Medicine and Public Health and Hunter Medical Research Institute, Newcastle, New South Wales, Australia ${ }^{2}$ Department of Respiratory and Sleep Medicine, John Hunter Hospital, New Lambton, New South Wales, Australia

\section{Correspondence to} Rebecca Louise Forbes, Department of Respiratory Medicine, Locked Bag 1, Hunter Region Mail, New Lambton, NSW, Australia, 2350; rebecca.I.forbes@uon.edu.au

Received 30 January 2011 Accepted 11 August 2011 Published Online First 13 September 2011

\title{
Impaired type I and III interferon response to rhinovirus infection during pregnancy and asthma
}

\author{
Rebecca Louise Forbes, ${ }^{1}$ Peter G Gibson, ${ }^{1,2}$ Vanessa E Murphy, ${ }^{1}$ Peter A B Wark ${ }^{2}$
}

\begin{abstract}
Background Acute respiratory tract infections are common ailments to all individuals and the human rhinoviruses (HRVs) cause most of these infections. Pregnant women have increased susceptibility and disease severity to viral infections like influenza and HRVs, as do asthmatics. Successful pregnancy requires immunological modulation to permit fetal tolerance.

Objectives To determine whether pregnant women have reduced innate antiviral interferon (IFN) responses to HRV infection compared with non-pregnant women. Methods An in vitro culture system was used, where peripheral blood mononuclear cells (PBMCs) were isolated from whole blood of 54 women, including 10 stable asthmatics who were pregnant and 10 who were not pregnant, 10 non-asthmatic women who were pregnant, 10 who were $\geq 6$ months post partum and 10 who were not pregnant. Samples were also collected from four exacerbating pregnant asthmatics. PBMCs were cultured with HRV43 and HRV1B. The antiviral proteins IFN $\alpha$ and IFN $\lambda$ were measured from culture supernatants by ELISA.
\end{abstract}

Results Compared with healthy non-pregnant women, pregnant women had significantly reduced innate IFN responses to HRV infection $(\mathrm{p}<0.02)$, persisting $\geq 6$ months post partum ( $\leq 00.02$ ). Pregnant asthmatics had significantly reduced IFN $\lambda$ responses compared with healthy non-pregnant women ( $p \leq 0.034)$, while during current asthma exacerbations a decrease in IFN $\alpha$ $(p \leq 0.023)$ and IFN $\lambda(p=0.007)$ was observed. Induction by a TLR7 agonist induced a similar pattern of decreased innate IFNs during pregnancy as observed when HRV was the inducing agent.

Conclusions Reduced antiviral IFNs during pregnancy and asthma provide an important mechanism for increased susceptibility, morbidity and mortality in pregnant women with respiratory viral infection.

\section{INTRODUCTION}

Respiratory virus infections are a major issue during pregnancy, since pregnant women have increased susceptibility to respiratory virus infections as well as subsequent increased disease severity and duration of infection. ${ }^{12}$ Pregnancy can be viewed as an 'immunological balancing act' where fetal tolerance (via maternal immunosuppression) is essential and yet a degree of maternal protection must be maintained against invading pathogens. Alterations in adaptive immunity have been identified during pregnancy; the most notable being a switch from T-helper-1 immunity towards a T-helper-2 type response. ${ }^{3}$ The innate immune system is essential

\section{Key messages}

What is the key question?

- What are the innate antiviral immune alterations in pregnancy and asthma that contribute to their increased susceptibility and disease severity to respiratory virus infection?

\section{What is the bottom line?}

- In pregnancy and asthma, there is a significant reduction in host antiviral type I and III interferons in response to human rhinovirus infections

Why read on?

- The results of this research are novel and provide us with an important insight as to why pregnant women are more susceptible and have increased disease severity to respiratory virus infections

in the host's defence during viral infections with the type I $(\alpha / \beta)$ and type III $(\lambda)$ interferons (IFNs) known to be a critical component of this innate defence. IFNs are released by virus-infected epithelial cells and peripheral blood mononuclear cells $(\mathrm{PBMCs})^{4}$ and act to facilitate both early innate immunity as well as priming the later antiviral adaptive immune response. ${ }^{5}$ Alterations in the innate immune response during pregnancy may have important implications; however, little research has investigated modifications that are likely to arise, and much less the potential effects this may have on immunity during viral infections.

Asthma is a common comorbidity during pregnancy with $18-36 \%$ of pregnant asthmatics reported to experience at least one severe asthma exacerbation requiring hospitalisation, emergency department presentation, unscheduled doctor's visit or a course of oral corticosteroids (OCS).? Human rhinoviruses (HRVs) are responsible for $60-80 \%$ of all respiratory virus-induced asthma exacerbations. ${ }^{8} 9$ Pregnant asthmatics have increased susceptibility to viral infections compared with pregnant non-asthmatics, ${ }^{10}$ as well as increased symptom severity and duration of infections compared with non-asthmatics. ${ }^{11} 12$

Blood and bronchial epithelial cells from nonpregnant asthmatics exhibit reduced antiviral type I and III IFN responses to respiratory viruses, associated with increased viral replication, airway inflammation and increased asthma symptoms. ${ }^{13-15}$ 
While asthmatics exhibit reduced IFN production to respiratory virus infections, it is not known whether pregnant women have a similar reduction or whether asthma in pregnancy further exaggerates this effect, thereby explaining the increased susceptibility observed in pregnant women during respiratory virus infections. We hypothesised that pregnant women would exhibit reduced type I and III IFN responses to in vitro HRV infection, which may be further worsened in asthmatics.

\section{MATERIALS AND METHODS Subjects}

This cross-sectional study of 54 participants included six groups: 10 pregnant women with asthma and 10 without, 10 nonpregnant women with asthma and 10 without, as well as 10 non-asthmatic women in the postpartum period ( $\geq 6$ months after childbirth) and 4 pregnant asthmatics with current asthma exacerbation (table 1). Pregnant women were recruited from the antenatal clinics at the John Hunter Hospital, as part of a larger randomised controlled trial (the 'Managing asthma in pregnancy' study ${ }^{16}$ ), while non-pregnant women were recruited from the Hunter Medical Research Institute Register and John Hunter Hospital respiratory clinics and staff (online supplement). Ethics approval was obtained from the Hunter New England Human Research Ethics Committee and the University of Newcastle Research Ethics Committee and informed consent was obtained from all subjects prior to participation.

\section{Design}

Baseline characterisation included height, weight, lung function, smoking status, medication and asthma history. Inclusion criteria for all participants were female gender, aged 18-40 years and currently non-smokers. Pregnant women were recruited between 18 and 28 weeks gestation. Asthmatics had a doctor's diagnosis of asthma, current asthma symptoms and/or asthma medication use. Current and retrospective cold and flu symptoms were assessed using the Common Cold Questionnaire (CCQ) classifying subjects as (1) 'no virus', (2) 'possible' or (3) 'probable', based on cold/flu symptom score (eg, nasal, chest and throat symptoms). ${ }^{17}$

For asthmatics, characterisation also included assessment of current therapy and symptoms, OCS use, and asthma control using the Asthma Control Questionnaire (ACQ) and asthma control criteria from the Global Initiative for Asthma (GINA) guidelines. ${ }^{18}$ Asthma exacerbations were defined as hospital admissions, unscheduled doctor's visits, \% forced expiratory volume in one second $\left(\mathrm{FEV}_{1}\right)<80 \%$ predicted, loss of control (ACQ and GINA criteria) or OCS within the last 2 weeks. Blood samples were collected within 2 days from the onset of exacerbation. Women were excluded if they had concomitant chronic medical illness or lung disease (besides asthma), drug or alcohol dependence, inability to perform spirometry or cold/flu symptoms within 1 month prior to sample collection.

\section{Viral stocks}

HRV43, a major group rhinovirus, was a clinical isolate obtained in 2005 and HRV1B, a minor group rhinovirus, was a gift from the Woolcock Institute of Medical Research in 2005. Viral stocks were propagated in RD-ICAMs (ATCC, Manassas, Virginia, USA), similar to that described previously. ${ }^{19}$ Viral concentration was determined by tissue culture infective dose $50 \%$, calculated using the Spearman-Karber formula. UV-inactivated virus was prepared as a negative control (online supplement).

\section{PBMC isolation and culture}

Whole blood was collected in EDTA tubes and PBMCs were isolated by density centrifugation using Ficoll-Paque PLUS (GE Healthcare Uppsala, Sweden), as per the manufacturer's instructions. PBMCs were resuspended in Roswell Park Memorial Institute media (Invitrogen, Australia Pty Limited), 5\% fetal bovine serum (SAFC Biosciences, Lenexa, Kansas, USA) and plated at a final concentration of $2.0 \times 10^{6}$ cells $/ \mathrm{ml}$ in 24 -well plates (sterile, tissue culture grade, NUNC, Denmark). Virus (live or UV-inactivated) was added at a multiplicity of infection 20, while the TLR3 agonist, polyinosine-polycytidylic acid (Poly (I:C); Sigma-Aldrich, Saint Louis, Missouri, USA), was used at a final concentration of $10 \mu \mathrm{g} / \mathrm{ml}$ and the TLR7 agonist, imiquimod (Invivogen, San Diego, California, USA), at a final concentration of $5 \mu \mathrm{g} / \mathrm{ml}$. PBMCs were cultured with virus or TLR agonists for a total of $48 \mathrm{~h}$, at $33^{\circ} \mathrm{C}$ and $5 \% \mathrm{CO}_{2}$. No media changes were made during this period. Cellular suspensions were centrifuged at $550 \times \mathrm{g}, 10 \mathrm{~min}$ and supernatants stored at $-80^{\circ} \mathrm{C}$ for subsequent analyses.

\section{Cell viability}

Cell viability was assessed routinely by Trypan-blue exclusion, which discriminates live from dead cells based on cellular necrosis. Cell viability was also tested in optimisation experiments using PE Annexin V Apoptosis Kit I (BD Bioscience California, USA), as per manufacturer's instructions. This method uses annexin and 7AAD to determine apoptotic (annexin ${ }^{+}$and $7 \mathrm{AAD}^{+}$) and necrotic (annexin ${ }^{-}$and $7 \mathrm{AAD}^{+}$) from viable cells (annexin ${ }^{-}$and $7 \mathrm{AAD}^{-}$). PBMCs consistently showed $>90 \%$ viability using both methods, when cultured with HRV or TLR agonists, as well as in media alone (online supplement).

Table 1 Characteristics of study groups

\begin{tabular}{llllll}
\hline Status & HC & P & PP & A & P A \\
\hline N & 10 & 10 & 10 & 10 & 10 \\
Age (years) & $29.7 \pm 7.2$ & $29.7 \pm 5$ & $29 \pm 4.3$ & $26.5 \pm 7.9$ & $27.4 \pm 6.1$ \\
Gestational age (weeks) & NA & $26.2 \pm 2.1$ & NA & NA & $25.1 \pm 1.9$ \\
ACQ & NA & NA & NA & $0.71(0.71)$ & $0.35(0.72)$ \\
\%FEV & $109.6 \pm 11.8$ & $101.4 \pm 8.5$ & $98.7 \pm 9.7$ & $92.8 \pm 9.1^{*}$ & $88.2 \pm 11.1^{*}$ \\
Blood IgE (IU/ml) & nd & $39.5(47)$ & $39(117)$ & nd & $289(384)^{*}$ \\
ICS use (n) & NA & NA & NA & 2 & 5 \\
ICS daily dose ( $\mu$ g/day BUD equivalent) & NA & NA & NA & $0(500)$ & $200.5(246.5)^{*}$ \\
\hline
\end{tabular}

Parametric data represented as mean \pm SD, non-parametric data as median (IQR).

$\mathrm{ACQ}$, a measure of asthma control based on the asthma control questionnaire, where an ACQ score of $>1.5$ is considered to be uncontrolled asthma; \% $\mathrm{FEV}$, forced expiratory volume in $1 \mathrm{~s}$ as per cent predicted; Blood IgE, total allergy score; IU, international units; ICS use, number of women on inhaled corticosteroids at the time of sample collection; ICS daily dose, total $\mu \mathrm{g} /$ day of budesonide (BUD) equivalents. Asthmatics were currently on budesonide-eformoterol (Symbicort), budesonide or fluticasone-salmeterol (Seretide), where $0.5 \mu \mathrm{g}$ fluticasone propionate $=1 \mu \mathrm{g}$ budesonide; nd, no data were collected; NA, not applicable; HC, healthy control (non-pregnant, non-asthmatic); P, pregnant; PP, post partum; A, asthma; PA, pregnant asthmatic; PA+X, pregnant asthmatic with current asthma exacerbation.

${ }^{*} \mathrm{p}<0.001$. 
Figure 1 Interferon- $\alpha$ (IFN $\alpha)$ and IFN $\lambda$ responses of peripheral blood mononuclear cells (PBMCs) from pregnant women to in vitro human rhinovirus (HRV) stimulation. Isolated PBMCs from pregnant (P), postpartum (PP) and non-pregnant healthy control $(\mathrm{HC})$ women were stimulated with HRV43 (A) or HRV1B (B). IFN $\alpha$ and IFN $\lambda$ protein concentrations $(\mathrm{pg} / \mathrm{ml})$ are shown on the left and right y-axes, respectively. Values are represented as median (IQR).
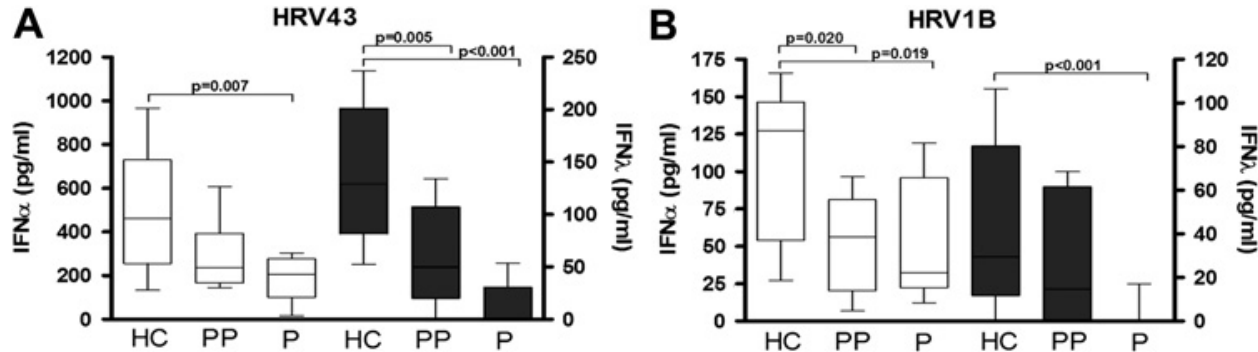

\section{ELISAs}

IFN $\alpha$ and IFN $\lambda$ protein concentrations were measured from culture supernatants by ELISA, as per the manufacturer's instructions, and analysed on a Fluorostar Optima microplate reader (BMG Labtech). The assay range for IFN $\alpha$ (PBL Interferon Source, New Jersey, USA) was $12.5-500 \mathrm{pg} / \mathrm{ml}$ and for IFN $\lambda$ (R\&D Systems, Minnesota, USA) $15.6-1000 \mathrm{pg} / \mathrm{ml}$. The minimal detectable dose was $\leq 10 \mathrm{pg} / \mathrm{ml}$. The inter- and intraassay variation was $8 \%$. The $\% \mathrm{CV}$ between duplicate samples was accepted when $\leq 5 \%$.

\section{PCR}

RNA collected from nasal and throat swabs was used for detection of respiratory viruses by two-step PCR. Briefly, cDNA was transcribed using the High-Capacity cDNA Reverse Transcription Kit (Applied Biosystems, Carlsband, California, USA) followed by quantitation of target mRNA with the TaqMan Gene Expression Master Mix (Applied Biosystems) and virus-specific primers (online supplement).

\section{Statistical analysis}

Statistical analyses were performed using STATA11 (Stata Corp). For subject statistics, the Kwallis2 test was conducted to determine significant differences in characteristics between groups. For asthmatic groups, correlations between the IFN responses and \%FEV or ACQ scores were tested using Kendall's $\tau$. The rank-sum test was used to assess differences in IFN response with inhaled corticosteroid (ICS) use in asthmatics. Simple linear regression was performed on asthmatic groups to determine if ICS dose was a determinant of IFN response. To determine if the study had sufficient power, the PS program (version 2.1.31) was used. ${ }^{20}$ For experimental statistics, significant differences between the groups were determined using the Kwallis2 test. Rank-sum tests were then conducted, comparing the healthy controls with each of the subsequent five groups (ie, $\mathrm{P}, \mathrm{A}, \mathrm{PA}, \mathrm{PP}$ and $\mathrm{PA}+\mathrm{X})$. Significance was accepted when $\mathrm{p}<0.05$ (online supplement). All protein data and figures were reported as median (IOR).

\section{RESULTS}

The women in this study had a mean age of $28.6 \pm 5.6$ years, with no significant difference between the groups (table 1). Pregnant women were recruited mid to late third trimester, averaging 26.6 \pm 3.2 . Asthmatics (pregnant and non-pregnant) had well-controlled asthma at the time of sample collection (ACQ scores <1.5), while currently exacerbating pregnant asthmatics showed loss of control with a significantly increased median ACQ score of 1.9 (0.95), $(\mathrm{p}<0.001)$. All asthmatic groups showed significantly lower \%FEV and significantly higher blood IgE levels compared with the non-asthmatic groups $(p<0.001)$. There was no significant difference in ICS doses between asthmatics. No subjects were taking OCS at the time of sample collection. No significant difference was seen in virus-induced IFN responses between asthmatics (pregnant and non-pregnant) currently on ICS treatment compared with those who were not and ICS dose was not a determinant of IFN response $(p>0.10)$.

\section{PBMCs from pregnant women have deficient IFN $\alpha$ and IFN $\lambda$ responses to in vitro HRV infection}

PBMCs from pregnant women stimulated with HRV43 (figure 1A) produced significantly less IFN $\alpha$ (median 206.5 (IOR 135.9), $\mathrm{p}=0.007)$ and IFN $\lambda(0,(28.8), \mathrm{p}<0.001)$ compared with PBMCs from healthy women (460.8 (193.8) and 136.4 (77.7), respectively). Similarly, in response to the minor group HRV1B (figure $1 \mathrm{~B}), \mathrm{PBMCs}$ from pregnant women produced significantly less IFN $\alpha$ (33.1 (70.5), $\mathrm{p}=0.019)$ and IFN $\lambda(0$ (5.4) $\mathrm{p}<0.001)$ compared with PBMCs from healthy non-pregnant women (127 (90.8) and 33.6 (59.3), respectively). To determine if this reduced IFN response persisted following pregnancy, PBMCs were isolated from women, 6-8 months post partum. HRV43-stimulated PBMCs from postpartum women (figure 1A) produced significantly less IFN $\lambda$ compared with PBMCs from non-pregnant women $(p=0.005)$. In response to HRV1B, there was significantly less IFN $\alpha$ produced by PBMCs from postpartum women compared with PBMCs from non-pregnant women $(p=0.020)$.

\section{TLR7 stimulation of PBMCs follows the same pattern as HRV- induced IFN $\alpha$ deficiency in pregnancy}

Stimulating the TLR7 pathway (figure 2) induced the same pattern of IFN $\alpha$ response observed for live virus with PBMCs from pregnant women producing significantly less IFN $\alpha$ compared with PBMCs from healthy non-pregnant women $(p=0.015)$. Stimulating the TLR3 pathway in PBMCs (online supplement) induced no significant difference in IFN response between the groups $(p>0.05)$.

\section{Asthma during pregnancy leads to reduced IFN $\lambda$ response to HRV infection}

There was no significant difference in HRV43-induced IFNa production in PBMCs obtained from asthmatics (307.3 (183.2)) or pregnant asthmatics (340.6 (326.7)) compared with PBMCs from non-pregnant women (460.8 (193.8)) (figure 3A). No significant difference was observed in IFN $\lambda$ response from PBMCs of asthmatics (81.2 (95.6)) compared with cells of healthy non-pregnant women (136.4 (77.7)); however, a significant reduction in IFN $\lambda$ was observed in PBMCs from pregnant asthmatics (46.4 (81.9), $p=0.012$ ). In response to HRV1B (figure $3 \mathrm{~B})$, a similar pattern was observed, with PBMCs from pregnant asthmatics producing significantly less IFN $\lambda$ (6.33 (24.6)) compared with PBMCs from healthy non-pregnant women (33.6 (59.3), $\mathrm{p}=0.034)$. TLR7 stimulation of PBMCs from pregnant asthmatic women (online supplement) induced a similar IFN response as live virus. 


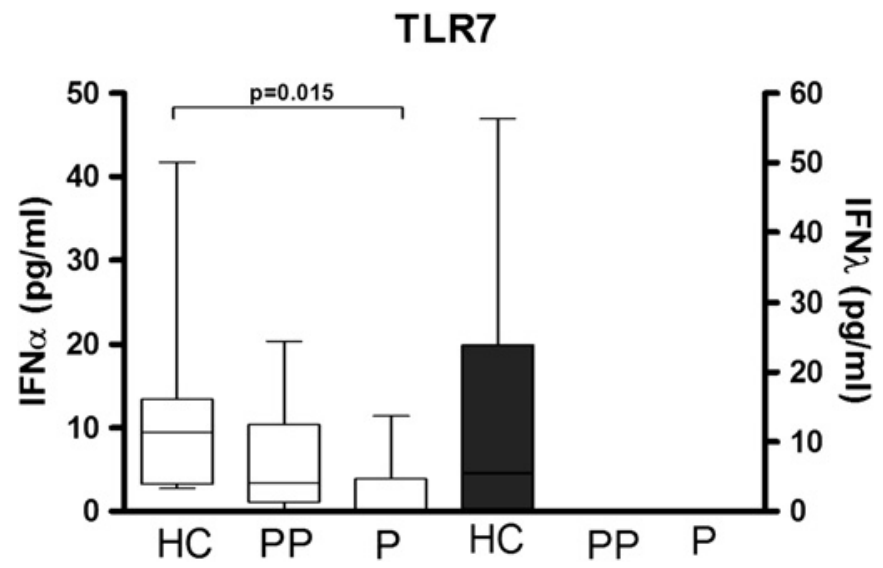

Figure 2 Interferon- $\alpha$ (IFN $\alpha$ ) and IFN $\lambda$ responses of peripheral blood mononuclear cells (PBMCs) to TLR7 agonists. Isolated PBMCs from pregnant $(\mathrm{P})$, postpartum (PP) and non-pregnant healthy control $(\mathrm{HC})$ women were stimulated with the TLR7 agonist imiquimod. IFN $\alpha$ and IFN $\lambda$ protein concentrations $(\mathrm{pg} / \mathrm{ml}$ ) are shown on the left and right $y$-axes, respectively. Values are represented as median (IOR).

\section{Pregnant asthmatics with an in vivo exacerbation have deficient type I and III IFN responses to in vitro HRV infection}

To see how PBMCs from pregnant women responded to in vitro rhinovirus infection during in vivo respiratory illness, PBMCs were collected from four pregnant asthmatics with cold/flu symptoms (ie, 'probable virus' score according to the CCQ) and current (within 2 days of onset) asthma exacerbations. All four women had ACQ scores $>1.5$ and showed loss of asthma control by GINA criteria (table 1). None required ED presentation or hospital admission, but all four had an unscheduled visit to their general practitioner or the respiratory research clinician. One woman had a \%FEV below $80 \%$ and two were prescribed a course of OCS following their doctor's visit (commenced after study sample collection). Exacerbations were considered respiratory virus-induced, as all scored 'probable' to the CCO. PCR analyses of nose and throat swabs during exacerbation confirmed one positive for human metapneumovirus infection and another for coronavirus.

In response to in vitro HRV43 infection (figure 4A), PBMCs from exacerbating pregnant asthmatics produced significantly less IFN $\alpha$ (135.5 (183), $p=0.023)$ and IFN $\lambda$, (23.9 (38.4), $\mathrm{p}=0.007)$ compared with PBMC responses from healthy nonpregnant women (460.8 (193.8) and 136.4 (77.7), respectively). HRV1B-induced IFN $\alpha$ (figure 4B) was also significantly lower in PBMCs from exacerbating pregnant asthmatics (19.6 (39.8), $\mathrm{p}=0.010)$ compared with the response from healthy non-pregnant cells $(127.7$ (90.8)). No significant correlations were found between ACQ score or FEV and the IFN responses of PBMCs from all asthmatic groups during in vitro HRV infection.

\section{DISCUSSION}

This study shows that pregnant women have deficient type I and III antiviral IFN responses to rhinovirus infection compared with healthy non-pregnant women. A similar pattern of reduced IFNs was observed in PBMCs of pregnant women when stimulated with a TLR7 agonist. A consistent and significant reduction in IFN $\lambda$ was seen in pregnant asthmatics in response to HRV and both IFN $\alpha$ and IFN $\lambda$ were significantly reduced in response to HRV in pregnant women during asthma exacerbations. These results indicate innate immune dysfunction as a possible mechanism for the increased susceptibility of pregnant women to respiratory virus infections.

Our data add to current knowledge by demonstrating that PBMCs from pregnant women showed a marked decrease in innate IFNs in response to both HRV43 and HRV1B. The regulation and importance of type I IFNs during pregnancy, especially in response to respiratory virus infection, is still unclear, while the role of type III IFNs has yet to be explored. Epidemiological studies have identified pregnant women as having increased susceptibility to respiratory virus infections ${ }^{21} 22$ and the HRVinduced reduction of IFN $\alpha$ and IFN $\lambda$ we observed in PBMCs from pregnant compared with non-pregnant women demonstrates an important mechanism explaining why pregnant women are more susceptible.

In response to both strains of $\mathrm{HRV}$, IFN $\alpha$ and IFN $\lambda$ production from PBMCs of postpartum women was significantly lower than that from PBMCs of non-pregnant women. This suggests a delay in returning to the 'normal' non-pregnant state, confirming heightened risk for respiratory viral infections in the postpartum period. ${ }^{23}$

TLR7, an ssRNA pathogen recognition receptor of the innate immune system, ${ }^{24}$ plays an important role in the type I and III IFN responses observed in this study. IFN $\alpha$ and IFN $\lambda$ were most markedly reduced in TLR7-stimulated PBMCs from pregnant (asthmatic and non-asthmatic) and postpartum women, indicating pregnancy-associated immune alterations as the most important factor altering TLR7 function. Considering TLR7 is a key receptor for sensing viral RNA, alteration in TLR7 may be an important mechanism for reduced IFNs during respiratory virus infection in pregnancy. Plasmacytoid dendritic cells ( $\mathrm{pDCs}$ ) are key producers of both IFN $\alpha$ and IFN $\lambda$ and abundantly express TLR7, ${ }^{25-29}$ making them important targets for future studies. TLR7 alterations may be due to reduced numbers of TLR7-expressing cells like pDCs or a change in receptor activity. Oestrogen is known to increase during pregnancy and has been found to alter $\mathrm{pDC}$ activity and function, ${ }^{30}$ providing a plausible link among pregnancy, IFNs and TLR7 activity during respiratory virus infections.
Figure 3 Interferon- $\alpha$ (IFN $\alpha$ ) and IFN $\lambda$ responses of peripheral blood mononuclear cells (PBMCs) from asthmatic women to in vitro human rhinovirus (HRV) stimulation. Isolated PBMCs from non-pregnant healthy control (HC) women and asthmatics who were pregnant $(\mathrm{PA})$ and not pregnant $(A)$ were stimulated with HRV43 (panel A) or HRV1B (panel B). $\mathrm{IFN} \alpha$ and IFN $\lambda$ protein concentrations $(\mathrm{pg} / \mathrm{ml})$ are shown on the left and right $\mathrm{y}$-axes, respectively. Values are represented as median (IOR).
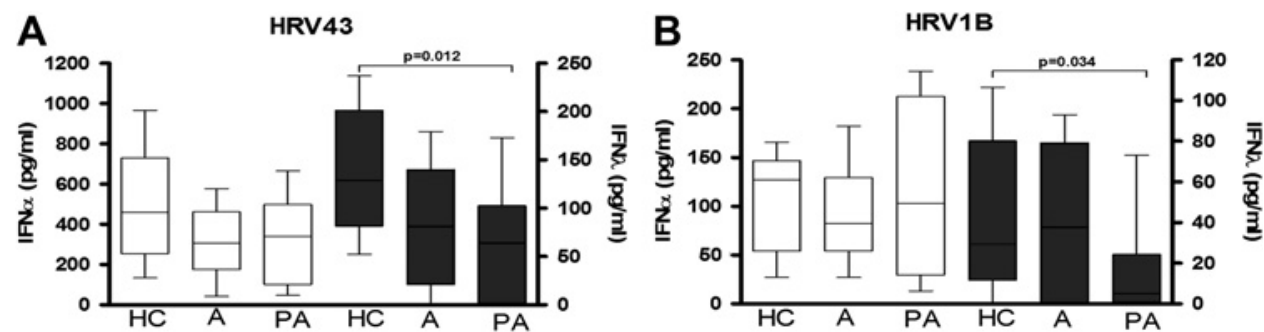
Figure 4 Interferon- $\alpha$ (IFN $\alpha)$ and IFN $\lambda$ responses of peripheral blood mononuclear cells (PBMCs) from asthmatic women to in vitro human rhinovirus (HRV) stimulation during in vivo exacerbation. Isolated PBMCs from pregnant asthmatics with current (in vivo) asthma exacerbations ( $\mathrm{PA}+\mathrm{X})$ were cultured (in vitro) with HRV43 (A) and HRV1B (B). IFN $\alpha$ and IFN $\lambda$ protein

A

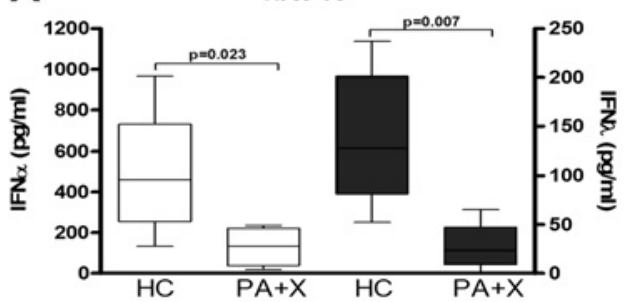

B

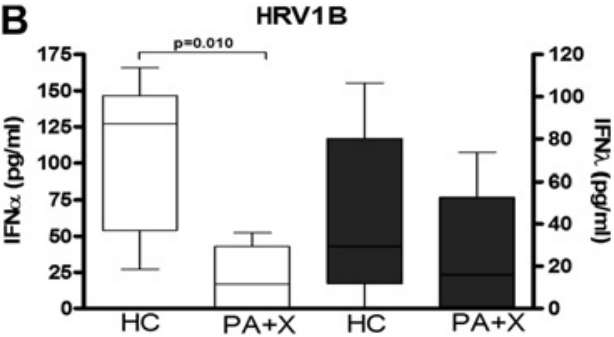
$\mathrm{PBMC}$ response from non-pregnant healthy control (HC) women. Values are represented as median (IQR).

This study shows that pregnancy, rather than asthma, appears to be the primary factor for a reduced innate IFN response. Systemic reduction of type I and III IFNs has been previously identified in asthmatic cells when stimulated with respiratory viruses. ${ }^{13-15}$ In our work, we did not observe a significant decrease in IFN production from HRV-stimulated PBMCs of asthmatics. This may be due to methodological differences in cell types used (whole blood, pBECs, BALF macrophages vs PBMCs), viral strains (RSV, HRV16 vs HRV43) and subject selection (male/female vs female only).

Several studies have reported increased susceptibility, symptom severity and duration of respiratory virus infections in pregnant asthmatics compared with pregnant nonasthmatics. ${ }^{10-12}$ We observed that in response to HRV43 and HRV1B, pregnant asthmatics showed significantly less IFN $\lambda$ production compared with healthy non-pregnant women. This may help explain the increased frequency of exacerbations in pregnant asthmatics that has been observed compared with non-pregnant asthmatics. ${ }^{31} 32$

In this study, we found that in vitro HRV infection of PBMCs from pregnant asthmatics currently experiencing an in vivo exacerbation resulted in a significantly lower innate IFN response compared with PBMCs from healthy non-asthmatic women. When using an in vitro method to assess antiviral responses from pregnant asthmatics, a more realistic result may be achieved if the PBMCs are isolated from asthmatics during a current asthma exacerbation, rather than when their asthma is well controlled. The exposure of PBMCs in asthmatics to high levels of inflammatory cytokines prior to isolation (as would typically occur during in vivo virus-induced asthma exacerbations ${ }^{33}$ ), and the decreased IFNs in PBMCs of pregnant asthmatics (as we have shown in this in vitro study), lead to a significant reduction of IFNs when pregnant asthmatics are currently exacerbating. This provides useful insight into how a pregnant asthmatic is likely to respond to concurrent respiratory viral infection when the asthma is uncontrolled, further highlighting the importance of well-controlled asthma, especially during pregnancy. The reduction of IFNs seen in pregnant asthmatics was not likely due to steroid use, as no significant association was identified between steroid dose and IFN production in PBMCs, which is similar to previous findings. ${ }^{14}$

There are several limitations to our study, including the relatively small sample size, with $n=10$ participants per group. While these numbers are comparable to other studies looking at cellular IFN responses to in vitro virus stimulation, ${ }^{13-15}$ the small numbers may explain lack of significance, especially for the asthma data. This may also prevent us from making generalisable statements about entire populations and may make it difficult to detect a true interaction between steroid use and IFN response. However, the study was adequately powered to detect the difference in IFN response in pregnancy compared with non-pregnant women and provides useful mechanistic evidence for the epidemiological evidence of increased susceptibility to infection in pregnancy.

\section{CONCLUSION}

Pregnant women are classified as a 'high risk' group for respiratory infections. Since IFNs play a critical role in viral defence, reduced levels of type I or III IFNs could render one at increased risk of developing infections and concurrent illness. This study demonstrated that in pregnant women the induction of both IFN $\alpha$ and IFN $\lambda$ was significantly reduced in response to HRV infection. This effect was observed for at least 6 months following childbirth providing an important insight into one possible mechanism whereby pregnant women are more susceptible to respiratory virus infections. Stimulating the TLR7 pathway in PBMCs induced a similar IFN response from PBMCs of pregnant women as observed by live HRV infection. Reduced type I and III IFNs in pregnant asthmatics, especially during current asthma exacerbations, also provide an explanation as to why pregnant asthmatics experience an increase in severe respiratory symptoms during virus infection. Understanding this alteration in innate immunity will enable the future development of effective target strategies aimed at IFN production, helping to prevent or at least control virus infection during pregnancy, thereby improving health outcomes for both the mother and the baby.

Acknowledgements Karen McLaughlin and Kelly Steel were the clinical nurses who helped with recruitment of the pregnant participants for this study. Patrick Macelduff was the statistician who provided input on the methods of data analyses.

Funding The National Health and Medical Research Council of Australia (NHMRC), Australia, and Australian Postgraduate Association (APA) PhD Scholarship, NSW, Australia, grant number 455593 and 455626 , to provide financial support required for recruiting, consumables, staff, etc, needed to undertake the research project.

Correction notice This article has been corrected since it was published Online First. The order of the authors has been updated.

\section{Competing interests None.}

Ethics approval Hunter New England Human Research Ethics Committee and the University of Newcastle Research Ethics Committee.

Contributors PGG, PABW and VEM were responsible for the planning and intellectual input/advice for the article. RLF was responsible for the planning, conduct and reporting of the work described in the article.

Provenance and peer review Not commissioned; externally peer reviewed.

\section{REFERENCES}

1. Jamieson DJ, Honein MA, Rasmussen SA, et al. H1N1 2009 influenza virus infection during pregnancy in the USA. Lancet 2009;374:451-8.

2. Widelock D, Csizmas L, Klein S. Influenza, pregnancy, and fetal outcome. Public Health Rep 1963;78:1-11.

3. Aris A, Lambert F, Bessette $P$, et al. Maternal circulating interferon- $\gamma$ and interleukin-6 as biomarkers of Th1/Th2 immune status throughout pregnancy. J Obstet Gynaecol 2008;34:7-11.

4. Khaitov MR, Laza-Stanca V, Edwards MR, et al. Respiratory virus induction of alphabeta- and lambda-interferons in bronchial epithelial cells and peripheral blood mononuclear cells. Allergy 2009;64:375-86. 
5. Biron CA. Role of early cytokines, including alpha and beta inteferons (IFN- $\alpha / \beta)$ in innate adaptive immune responses to viral infections. Immunology 1998;10:383-90.

6. Ank N, West H, Paludan SR. IFN-lambda: novel antiviral cytokines. J Interferon Cytokine Res 2006:26:373-9.

7. Schatz M, Dombroski MP, Wise R, et al. Asthma morbidity during pregnancy can be predicted by severity classification. J Allergy Clin Immunol 2003:112:283-8.

8. Nicholson KG, Kent J, Ireland DC. Respiratory viruses and exacerbations of asthma in adults. BMJ 1993;307:982-6.

9. Johnston SL, Pattermore PK, Sanderson G, et al. Community study of the role of viral infections in exacerbations of asthma in 9-11 year old children. BMJ 1995;310:1225-9.

10. Minerbi-Codish I, Fraser D, Avnun L, et al. Infuence of asthma in pregnancy on labour and the newborn. Respiration 1998;65:103-35.

11. Cox S, Posner SF, McPheeters M, et al. Hospitalizations with respiratory illness among pregnant women during influenza season. J Obstet Gynaecol 2006;107:1315-22.

12. Hartert TV, Neuzil KM, Shintani AK, et al. Maternal morbidity and perinatal outcomes among pregnant women with respiratory hospitilizations during influenza season. J Obstet Gynaecol 2003:189:1705-12.

13. Contoli M, Message SD, Laza-Stanca V, et al. Role of deficient type III interferon- $\lambda$ production in asthma exacerbations. Nat Med 2006;12:1023-6.

14. Gehlhar K, Bilitewski C, Reintz-Rademecher K, et al. Impaired virus-induced interferonalpha2 release in adult asthmatic patients. Clin Exp Allergy 2006;36:331-7.

15. Wark PA, Johnston SL, Bucchieri F, et al. Asthmatic bronchial epithelial cells have a deficient innate immune response to infection with rhinovirus. J Exp Med 2005;201:937-47.

16. McLaughlin K, Murphy V, McCaffery K, et al. The relationship between patient perceived risk of inhaled corticosteroids in pregnancy and medication adherence. Respirology 2009;14:A42.

17. Powell H, Smart J, Wood L, et al. Validity of the common cold questionnaire (CCO) in asthma exacerbations. PLOS ONE 2008;3:e1802.

18. Juniper EF, Bousquet J, Abetz L, et al. Identifying 'well-controlled' and 'not wellcontrolled' asthma using the Asthma Control Questionnaire Respir Med 2006; 100:616-21.

19. Papi $\mathbf{A}$, Johnston SL. Rhinovirus infection induces expression of its own receptor intercellular adhesion molecule 1 (ICAM-1) via increased NF-KB-mediated transcription. J Biol Chem 1999;274:9707-20.
20. Dupont WD, Plummer WD. PS power and sample size program for free on the Internet. Control Clin Trials 1997;18:274.

21. Dodds L, McNeil SA, Fell DB, et al. Impact of influenza exposure on rates of hospital admissions and physician visits because of respiratory illness among pregnant women. CMAJ 2007;176:463-8

22. Neuzil KM, Reed GW, Mitchel EF, et al. Impact of influenza on acute cardiopulmonary hospitalizations in pregnant women. Am J Epidemiol 1998; 148:1094-102.

23. Groer MW, Davis MW, Smith $\mathrm{K}$, et al. Immunity, inflammation and infection in post-partum breast and formula feeders. Am J Reprod Immunol 2005;54:222-31.

24. Yang K, Puel A, Zhang S, et al. Human TLR-7-, -8-, and -9- mediated induction of IFN- $\alpha / \beta$ and $-\lambda$ is IRAK-4 dependent and redundant for protective immunity to viruses. Immunity 2005:23:465-78.

25. Birmachu W, Gleason R, Bulbulian B, et al. Transcriptional networks in plasmacytoid dendritic cells stimulated with synthetic TLR 7 agonists. BMC Immunol 2007:8:26

26. Coccia E, Severa M, Giacomini E, et al. Viral infection and toll-like receptor agonists induce a differential expression of type I and $\lambda$ interferons in human plasmacytoid and monocyte-derived dendritic cells. Eur J Immunol 2004;34:796-805.

27. Gibson SJ, Lindh JM, Riter TR, et al. Plasmacytoid dendritic cells produce cytokines and mature in response to the TLR7 agonists, imiquimod and resiquimod. Cell Immunol 2002; 218:74-86.

28. Ito T, Wang Y-H, Liu Y-J. Plasmacytoid dendritic cell precursors/type I interferonproducing cells sense viral infection by Toll-like receptor (TLR) 7 and TLR9 Springer Semin Immunopathol 2005;26:221-9.

29. Lund JM, Alexopoulou L, Sato A, et al. Recognition of single-stranded RNA viruses by Toll-like receptor 7. Proc Natl Acad Sci U S A 2004;101:5598-603.

30. Li X, Xu Y, Ma L, et al. 17beta-estradiol enhances the response of plasmacytoid dendritic cell to CpG. PLoS ONE. 2009;4:e8412.

31. Asthma in Australia: findings from the 2004-05 National Health Survey2007. http:// www.aihw.gov.au/publications/index.cfm/title/10416 (accessed Jul 2009).

32. Murphy VE, Gibson P, Talbot PI, et al. Severe asthma exacerbations during pregnancy. J Obstet Gynaecol 2005;106:1046-54

33. Kelly JT, Busse WW. Host immune responses to rhinovirus: mechanisms in asthma. J Allergy Clin Immunol 2008;122:671-82.

\section{Journal club}

\section{A possible mechanism of drug tolerance in mycobacteria}

In this study, the authors investigated possible mechanisms of drug tolerance in mycobacteria and identified a key mediator to target with drug therapy, with the aim of shortening current treatment and moving towards eradication.

Using an in vivo model, they found that drug tolerant mycobacteria existed in macrophages prior to granuloma formation, indicating that the granuloma is not essential for developing tolerance, but is important for dissemination. By monitoring the movement of individual macrophages from a particular granuloma they observed that these macrophages could leave the granuloma and recruit other macrophages to create new granulomas, often at sites distant from the original. This explained a well-established phenomenon in human tuberculosis infection.

The group confirmed the importance of macrophages in the induction of drug tolerance and showed that efflux pumps are essential for both bacterial growth and development of drug tolerance. They found that bacteria retained intracellularly induced tolerance when they became extracellular. This explained how tolerant extracellular bacteria could arise in the granuloma's necrotic core in human tuberculosis infection.

By challenging current dogma, the researchers have established the important role of macrophages and granulomas in inducing and disseminating tolerance. They have found efflux pumps to be a useful specific target of drug therapy due to their role in tolerance and bacterial growth.

- Adams KN, Takaki K, Connolly LE, et al. Drug tolerance in replicating mycobacteria mediated by a macrophage-induced efflux mechanism. Cell 2011;145:39-53.

\section{S V Ranson}

Correspondence to S V Ranson, St Mary's Hospital, London, UK; svranson@googlemail.com

Provenance and peer review Not commissioned; internally peer reviewed.

Published Online First 31 July 2011

Thorax 2012;67:214. doi:10.1136/thoraxjnl-2011-200800 